\title{
Humus is a guarantor of the stability of agroecosystems
}

\author{
E.S. Gasanova*, A.N. Kozhokina, N.G. Myazin, and K.E. Stekolnikov \\ FSBEI HE Voronezh SAU n.a. Emperor Peter I, 394087, Voronezh, Russian Federation
}

\begin{abstract}
The paper studies the processes of transformation of the molecular structure of humic (HA) and fulvic acids (FA) of leached chernozem under the influence of fertilizers and meliorant. The research was carried out under the conditions of many years of stationary experiment of the Department of Agrochemistry, Soil Science and Agroecology of the FSBEI HE Voronezh SAU. Samples of leached low-humus thin heavyloamy chernozem were analyzed. The isolated and purified preparations of HA and FA were studied on a SM2203 spectrofluorimeter in the ultraviolet and visible ranges. The paper also presents the results of determining the most important physical and chemical properties of soil variants using various fertilizer and meliorant systems. Limed variants have optimal indicators. Based on the analysis, it can be assumed that the FA molecules of the variant with a double dose of mineral fertilizers on the background of manure differ in a developed aliphatic part, have many auxochromic substituents. HA of the same variant are characterized by a high condensation of nuclear structures. The HA and FA molecules of the variant with the joint application of fertilizers and meliorant have a more complex structure of the nuclear and peripheral parts. According to the results of calculations of extinction coefficients, it was found that liming contributes to the accumulation of more mature forms of humic acids.
\end{abstract}

\section{Introduction}

Since the second half of the XX century, scientists have noted a sharp increase in the rate of soil degradation, which leads to a decrease in the level of soil fertility. This problem is also acute in the CCR of the Russian Federation. Two of the most important negative consequences of soil degradation are a decrease in the humus content and acidification [4]. The reason for these problems is the loss of calcium by the soil, which performs many functions and is a guarantor of soil fertility. It contributes to the accumulation of mature, stable forms of humus, determines many physical and chemical properties of the soil, such as actual and potential acidity, the amount of exchange bases, the degree of soil saturation with bases. To optimize the state of organic matter and regulate soil acidity, many authors suggest to perform chemical reclamation - liming [5, 8]. As lime materials in our region, it is advisable to use the waste of sugar beet production - defecate.

\footnotetext{
*Corresponding author: upravlenieopm@mail.ru
} 


\section{Object of study and methodology}

The work was carried out at the station of the Department of Agrochemistry, Soil Science and Agroecology of the FSBEI HE Voronezh SAU. The scheme of the experiment is described in detail in [4]. Soil samples of leached chernozem layer 0-20 cm were used as objects of research. The following options were studied: 1 . Without fertilizers - control, 2.40 $\mathrm{t} /$ ha of manure, $3.40 \mathrm{t} / \mathrm{ha}$ of manure + NPK, $5.40 \mathrm{t} / \mathrm{ha}$ of manure $+2 \mathrm{NPK}, 12.40 \mathrm{t} / \mathrm{ha}$ of manure $+\mathrm{NPK}+$ defecate of $1.0 \mathrm{Ng}, 13.40 \mathrm{t} / \mathrm{ha}$ of manure + defecate of $1.0 \mathrm{Ng}, 15$. NPK + defecate of $1.0 \mathrm{Ng}$.

Preparations of humic acids were isolated by the Kononova-Belchikova method, followed by separation into $\mathrm{HA}$ and FA by acidification to $\mathrm{pH} 1.5$. Further, their ion exchange purification was carried out using anionite AV-17-8 and cationite KU-2. As a result, HA and FA were obtained in $\mathrm{H}^{+}$-form. Samples of humic acids were dried in air. The spectra of HA and FA solutions of different variants were recorded in the wavelength range of 200-800 nm with a step of $5 \mathrm{~nm}$ on a SM2203 spectrofluorimeter. Thus, the optical properties of the preparations in the ultraviolet and visible wavelength ranges were studied.

\section{Research results}

Table 1 presents the results of determining the main physical and chemical characteristics of the analyzed soil samples. It was found that liming carried out on 12, 13 and 15 variants contributed to the optimization of the values of exchange and hydrolytic acidity, an increase in the amount of exchange bases by $8.79-9.02 \%$ of the initial values.

Table 1. The effect of fertilizers and defecate on the physico-chemical properties of leached chernozem, average in a layer of $0-40 \mathrm{~cm}, 2018-2019$.

\begin{tabular}{|c|c|c|c|c|c|c|c|c|}
\hline \multirow{2}{*}{$\begin{array}{c}\text { Variants } \\
\text { of experiment }\end{array}$} & \multicolumn{2}{|c|}{$\mathrm{pHKCl}$} & \multicolumn{2}{c|}{$\begin{array}{c}\text { Ng, } \\
\text { mg-eq./100 g of soil }\end{array}$} & \multicolumn{2}{c|}{$\begin{array}{c}\text { S, } \\
\text { mg-eq./100 g of soil }\end{array}$} & \multicolumn{2}{c|}{ C total, \% } \\
\cline { 2 - 9 } & $1^{*}$ & $2 * *$ & 1 & 2 & 1 & 2 & 1 & 2 \\
\hline 1. & 4.9 & 4.8 & 5.1 & 5.3 & 23.7 & 23.3 & 2.35 & 2.43 \\
\hline 2. & 5.0 & 5.1 & 4.8 & 4.1 & 24.3 & 24.7 & 2.48 & 2.62 \\
\hline 3. & 4.9 & 5.0 & 6.0 & 4.1 & 23.6 & 24.4 & 2.86 & 2.69 \\
\hline 5. & 4.7 & 4.9 & 6.4 & 4.4 & 23.4 & 24.3 & 2.76 & 2.68 \\
\hline 12. & 5.5 & 6.6 & 4.2 & 0.9 & 24.8 & 27.2 & 3.31 & 3.27 \\
\hline 13. & 5.6 & 6.7 & 3.7 & 0.8 & 25.2 & 27.7 & 3.06 & 2.93 \\
\hline 15. & 5.4 & 6.1 & 4.2 & 1.1 & 24.9 & 27.3 & 2.96 & 2.93 \\
\hline
\end{tabular}

Note: $1^{*}$ - before application of manure and defecate (18.04.2018);

$2 * *$ - before winter wheat harvesting (17.07.2019)

The content of organic carbon in the studied soil variants decreased by $1.02-6.31 \%$ from the source state, with the exception of the control and the variant with the introduction of manure. In these variants, the increase was 0.08 and $0.14 \%$, respectively. It is of interest to calculate the correlation coefficient between the humus content and the sum of the exchange bases. It is 0.60 and 0.87 for 2018 and 2019, respectively, which indicates a high closeness of the relation between these indicators.

The obtained results allow to conclude that the additional introduction of calcium into the soil contributes to the improvement of the most important physical and chemical properties.

Figure 1 shows the electronic spectra on the example of the HA of the studied fertilization variants. FA are characterized by similar spectral curves, a detailed description of the characteristic stripes is presented in the text. 


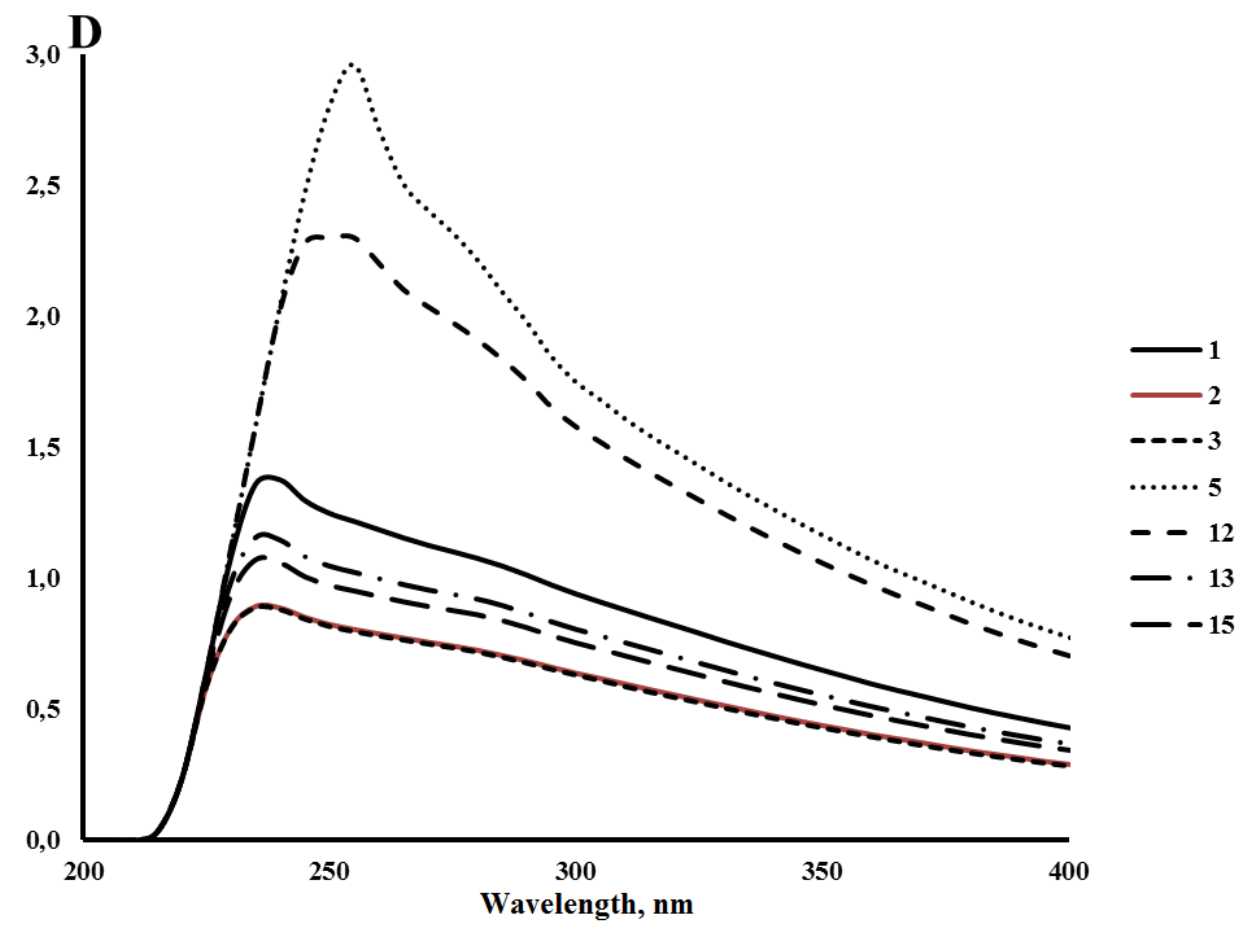

Fig. 1. Electronic spectra of HA of various variants.

To determine the exact position of the absorption maxima on such spectral curves, the dependence of the first derivative of the optical density on the wavelength is used under the condition $\mathrm{dD} / \mathrm{d} \lambda=0[5]$. The presented curves are characterized by a developed maximum in the range of $230-255 \mathrm{~nm}$, which is determined by benzene fragments with a developed auxochromic substituent, for example, with a carboxyl group [1]. There is also a certain jump in the region of $275 \mathrm{~nm}$, which is explained by the presence of phenolic compounds. On the spectra of variants with the introduction of a double dose of mineral fertilizers (option 5), as well as with the joint application of fertilizers and defecate (option 12), there is a sharp hyperchromic effect, as well as a bathochromic shift to the long-wave region of the spectrum. The optical density values at a wavelength of $255 \mathrm{~nm}$ are 2.92 and 2.27 for 5 and 12 variants, respectively. This can characterize a large amount of phenylcarboxylic acids in the HA molecules. It can be assumed that the HA molecules of these variants have developed nuclear and aliphatic structures. The reasons for this may be as follows. When applying high doses of mineral fertilizers (option 5), the intensity of hydrolysis and oxidation processes in soils increases, which is confirmed by the literature data [3]. Therefore, it can be assumed that the HA molecules of the 5 variant will be more condensed as a result of the transformation of the peripheral carbohydrate part. In case of joint application of fertilizers and calcium meliorant, the degree of condensation of molecules increases as a result of the accumulation of mature HA associated with calcium [4].

Thus, it can be assumed that the applied fertilizers and defecate change the molecular structure and the ratio of nuclear and peripheral fragments of HA molecules.

It is revealed that the electronic spectrum of the FA of the control variant is characterized by a maximum at $235 \mathrm{~nm}$ and an inflection at $280 \mathrm{~nm}$. It is established that some variants are characterized by a similar course of spectral curves. This applies to the background version, 
as well as options with the introduction of a single dose of mineral fertilizers on an organic background (option 3), as well as with the use of mineral fertilizers together with liming. It is worth noting that at $230 \mathrm{~nm}$ there is an increase in the optical density, and in the longer wavelength part of the spectrum a gentle course of curves is seen.

FA variants with the introduction of a double dose of mineral fertilizers on an organic background (option 5) have a sharply different type of electronic spectrum. Attention is drawn to the bathochromic shift of the maximum to $255 \mathrm{~nm}$, a bend in the region of $280 \mathrm{~nm}$ is highlighted. In addition, there is a sharp increase in the optical density of the solution to $\mathrm{D}_{255}=3$ (hyperchromic effect). The spectrum of the FA variant with the combined use of mineral, organic fertilizers and defecate (option 12) is complex. It is characterized by the presence of maxima at $235,250 \mathrm{~nm}$, as well as an inflection at $280 \mathrm{~nm}$. The FA spectrum of the defecated variant (variant 13) has a standard curve course with a maximum in the region of $235 \mathrm{~nm}$ at an optical density of 1.112; no peaks are observed in the bathochromic region.

Based on the analysis, it can be assumed that the FA molecules of the variant with a double dose of mineral fertilizers on the background of manure (variant 5) differ in a developed aliphatic part, have many auxochromic substituents. The FA molecules of the variant with the joint application of fertilizers and meliorant have a more complex structure of the nuclear and peripheral parts.

The visible wavelength range is not considered by many authors, as it is considered uninformative. Nevertheless, based on the analysis of the spectrum of humic acids in the range of 400-800 nm, several important characteristics of molecules can be calculated.

Figures $2 \mathrm{a}$ and $2 \mathrm{~b}$ show the spectra of HA and FA preparations in the visible wavelength range. The smoothly decreasing course of the curves in the range of 400-800 nm corresponds to the known literature data [7]. There are no bends and maximums. For the analysis of this area, it is proposed to use the extinction coefficient E465/E650. Rigobello et al. [6] reported that the E4/E6 ratio is influenced by the size of the molecule, the $\mathrm{pH}$ of the environment, the levels of oxygen, carbon and carboxyl groups, as well as the age of the humic material. This affects the formation of humic substances, which, according to Stevenson [2], occurs by three mechanisms: a) microbiological activity releases nitrogen-containing compounds that react with lignins; b) oxidation of polyphenolic compounds to form quinones, which are condensates in reactions with aminated compounds, and c) reduction of sugars that react with aminated compounds and turn into dark-colored macropolymers with high molecular weight. 

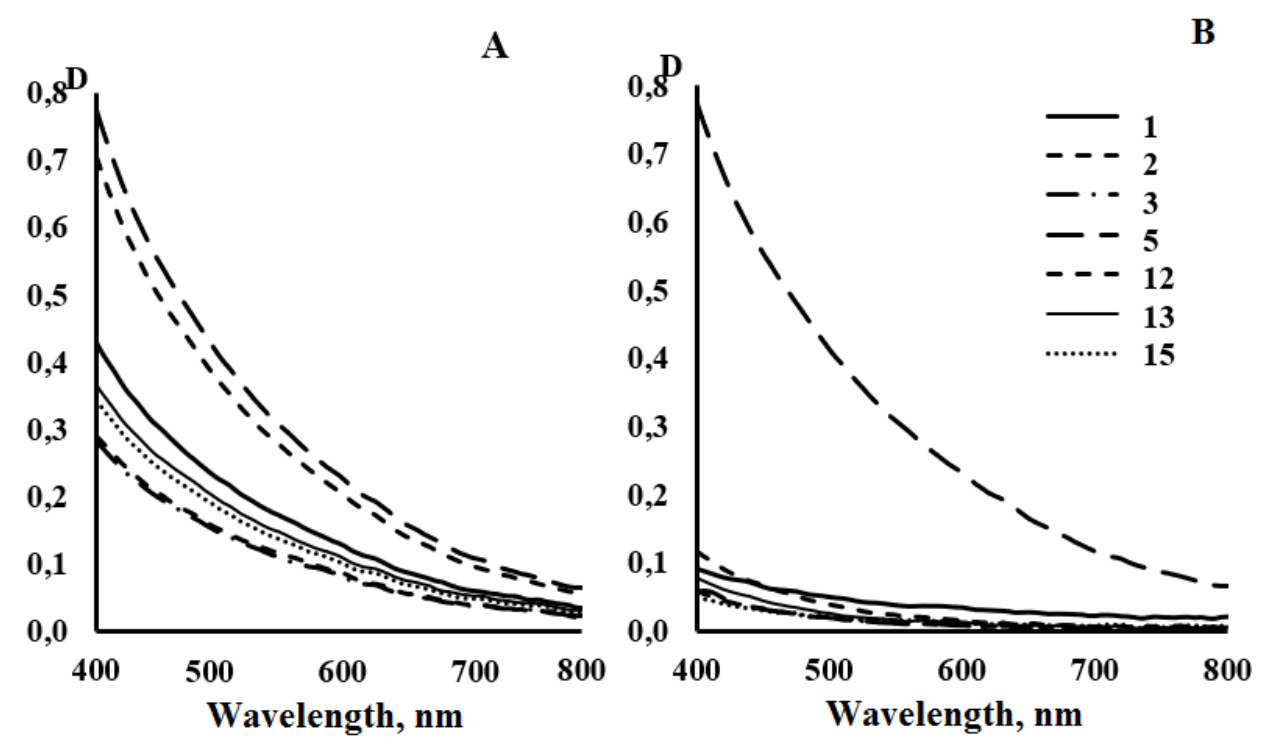

Fig. 2. Spectra of the analyzed samples of HA and FA.

A huge role in these processes is played by various enzymes released by soil microorganisms.

The results of the calculations are presented in the form of Table 2.

Table 2. Extinction coefficients of the HA and FA variants.

\begin{tabular}{|c|c|c|c|c|c|c|c|}
\hline E4/E6 & 1 var & 2 var & 3 var & 5 var & 12 var & 13 var & 15 var \\
\hline HA & 3.08 & 3.25 & 3.22 & 3.08 & 3.14 & 3.10 & 3.09 \\
\hline FA & 2.19 & 5.11 & 4.36 & 3.07 & 4.94 & 4.45 & 2.89 \\
\hline
\end{tabular}

The maximum values of the extinction coefficient for HA molecules are noted in the variants with the introduction of organic fertilizers - 3.25 (variant 2), as well as when using a single dose of mineral fertilizers on an organic background - 3.22 (variant 3). This may indicate the aliphatization of the HA molecules of these variants. Low indicators are typical for the control variant - 3.08, as well as for the variant using a double dose of mineral fertilizers -3.08 (variant 5). The established feature may be associated with the destruction of aliphatic structures of HA and an increase in their aromaticity. Defecated variants $(12,13$, 15 variants) are characterized by average indicators. It can be assumed that the use of meliorant contributes to the accumulation of HA molecules in the soil with developed nuclear and peripheral structures.

The highest values of E4/E6 calculated for FA molecules are noted on the background and limed variants: $2,12,13-5.11 ; 4.94 ; 4.45$ respectively. The minimum values are typical for the FA of the control variant (2.19) and the variant using a double dose of mineral fertilizers (3.07). The selection features can be explained as follows. In the background and reclaimed variants, during the preparative separation of humus, molecules with a predominance of aliphatic structures pass into the FA fraction. In the control variant and the variant with a high dose of NPK, the analyzed solution contains more condensed molecules. 
In his works, Zalfeld [8] recommends using the colority coefficients E400/E500, $\mathrm{E} 500 / \mathrm{E} 600, \mathrm{E} 600 / \mathrm{E} 700$. Table 2 shows the results of calculating the corresponding colority coefficients for HA preparations.

Table 3. Zalfeld colority coefficients for HA and FA.

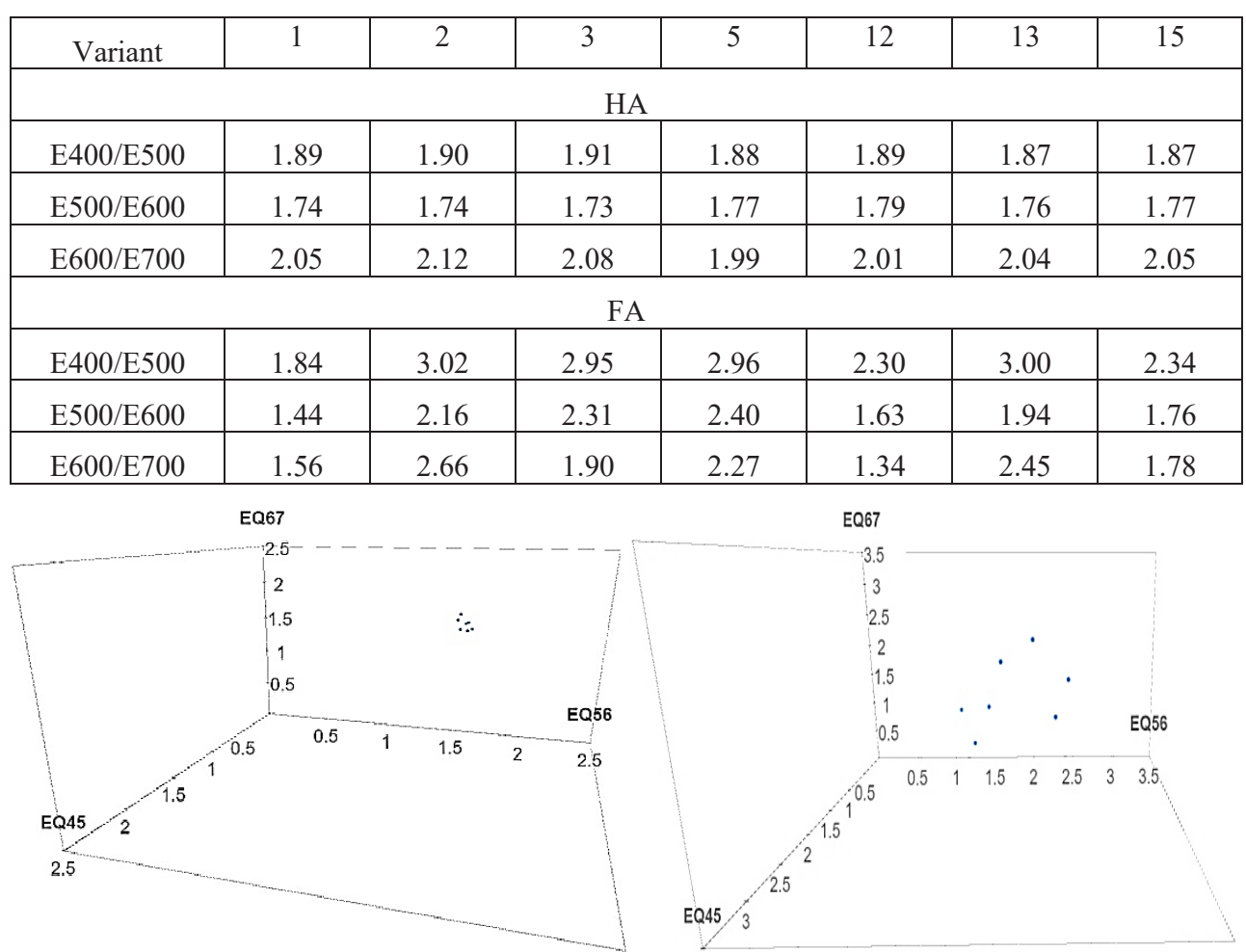

Fig. 3. Schematic model of the image of the optical density of HA and FA using three colority coefficients.

The obtained values are in a very narrow range from 1.73 to 2.12 . A similar range is typical for HA formed under the conditions of the sod soil-forming process. This indicates that, despite the long-term intensive various agricultural use, there have been no significant changes in the nature of HA.

The FA results indicate a completely opposite situation, since they are in a fairly wide range of values from 1.44 to 3.02, therefore, changes have occurred in the nature of FA. This is also determined by the nature of the structure of FA molecules, their mobility and high reactivity.

\section{Conclusions}

It is revealed that the studied agrotechnical techniques change many properties of soils, such as acidity, humus content and the amount of exchange bases. In addition, changes occur in the structure of humic acids. Using optical methods, we have established that various systems of applying fertilizers and meliorant lead to the transformation of the molecular structure of humic acids, changing the ratio of benzene rings and side aliphatic chains. Thus, liming leads to an increase in the number of highly condensed fragments of molecules with the preservation of a developed peripheral structure. The introduction of mineral fertilizers without the additional use of calcium meliorants not only worsens the physical and chemical 
properties of the soil, but also contributes to the destruction of aliphatic fragments of humus molecules.

Thus, based on the work carried out, we came to the conclusion that liming is necessary to compensate for the negative impact of high doses of mineral fertilizers, as well as to stabilize the most important component of any ecosystem - soil humus.

\section{References}

1. M. Banach-Szott, B. Debska, \& E. Tobiasova, Environmental Science and Pollution Research, (2021) doi:10.1007/s11356-021-14616-9

2. T. Cui, Z. Li, \& S. Wang, Journal of Soils and Sediments, 17(10), 2391-2399 (2017) doi:10.1007/s11368-017-1704-6

3. D.I. Eremin, J. Eurasian Soil Science 5, 538-545 (2016)

4. E.S. Gasanova, A. Kozhokina, N. Myazin, K.E. Stekolnikov, Bulletin of the Voronezh SAU 4 (59), 13-21 (2018)

5. V.V. Kotov, K.E. Stekolnikov, S.V. Tkachenko, S.V. Martynenko, E.S. Gridyaeva, J. Eurasian Soil Science 6, 618-623 (2004)

6. B.S. Ndzelu, S. Dou, \& X. Zhang, Soil Research, 58(5), 452-460 (2020) doi:10.1071/SR20025

7. B.S. Ndzelu, S. Dou, X. Zhang, Y. Zhang, R. Ma, \& X. Liu, Soil and Tillage Research, 213 (2021) doi:10.1016/j.still.2021.105090

8. N.E. Zav'yalova, J. Eurasian Soil Science, 6, 627-633 (2015) 